\title{
BMJ Open HOMESIDE: home-based family caregiver-delivered music and reading interventions for people living with dementia: protocol of a randomised controlled trial
}

\author{
Felicity Anne Baker (D , ${ }^{1}$ Jodie Bloska, ${ }^{2}$ Sabine Braat, ${ }^{3}$ Anna Bukowska, ${ }^{4}$ \\ Imogen Clark, ${ }^{1,5}$ Ming $\mathrm{H} \mathrm{Hsu},{ }^{2}$ Tone Kvamme, ${ }^{6}$ Nicola Lautenschlager, ${ }^{7,8}$ \\ Young-Eun Claire Lee, ${ }^{1,5}$ Agnieszka Smrokowska-Reichmann, ${ }^{4}$ \\ Tanara Vieira Sousa, ${ }^{3}$ Karette A Stensaeth, ${ }^{6}$ Jeanette Tamplin, ${ }^{1,5}$ Thomas Wosch, ${ }^{9}$ \\ Helen Odell-Miller ${ }^{2}$
}

To cite: Baker FA, Bloska J, Braat S, et al. HOMESIDE: home-based family caregiver-delivered music and reading interventions for people living with dementia: protocol of a randomised controlled trial. BMJ Open 2019;9:e031332. doi:10.1136/ bmjopen-2019-031332

- Prepublication history for this paper is available online. To view these files, please visit the journal online (http://dx.doi org/10.1136/bmjopen-2019031332).

Received 29 April 2019 Revised 02 August 2019 Accepted 25 October 2019

Check for updates

(C) Author(s) (or their employer(s)) 2019. Re-use permitted under CC BY-NC. No commercial re-use. See rights and permissions. Published by BMJ.

For numbered affiliations see end of article.

Correspondence to

Dr Felicity Anne Baker;

felicity.baker@unimelb.edu.au

\section{ABSTRACT}

Introduction Pharmacological interventions to address behavioural and psychological symptoms of dementia (BPSD) can have undesirable side effects, therefore nonpharmacological approaches to managing symptoms may be preferable. Past studies show that music therapy can reduce $B P S D$, and other studies have explored how formal caregivers use music in their caring roles. However, no randomised study has examined the effects on BPSD of music interventions delivered by informal caregivers (CGs) in the home setting. Our project aims to address the need for improved informal care by training cohabiting family CGs to implement music interventions that target BPSD, and the quality of life $(\mathrm{Q} o \mathrm{~L})$ and well-being of people with dementia (PwD) and CGs.

Methods and analysis $A$ large international three-arm parallel-group randomised controlled trial will recruit a sample of 495 dyads from Australia, Germany, UK, Poland and Norway. Dyads will be randomised equally to standard care (SC), a home-based music programme plus SC, or a home-based reading programme plus SC for 12 weeks. The primary outcome is BPSD of PwD (measured using the Neuropsychiatric Inventory-Questionnaire). Secondary outcomes will examine relationship quality between CG and PwD, depression, resilience, competence, QoL for CG and QoL for PwD. Outcomes will be collected at baseline, at the end of the 12-week intervention and at 6 months post randomisation. Resource Utilisation in Dementia will be used to collect economic data across the life of the intervention and at 6-month follow-up. We hypothesise that the music programme plus SC will generate better results than SC alone (primary comparison) and the reading programme plus SC (secondary comparison). Ethics and dissemination Ethical approval has been obtained for all countries. Results will be presented at national and international conferences and published in scientific journals and disseminated to consumer and caregiver representatives and the community. Trial registration numbers ACTRN12618001799246p; NCT03907748

\section{Strengths and limitations of this study}

- As a multinational trial, this study will provide internationally generalisable results concerning the effects of music interventions delivered by trained informal caregivers on the behavioural and psychological symptoms of people living with dementia.

- Based on pilot data, this trial will have adequate power to determine any effects on the person with dementia.

- The trial will determine whether caregiver-delivered music interventions improve caregiver quality of life and well-being, and reduce healthcare costs for the caregiver and society.

- A comprehensive set of core outcomes will be measured, including long-term effects in key variables, with assessor blinding.

- As participants cannot be blinded, a limitation of the study is that they may provide biased responses on self-report measures.

\section{BACKGROUND}

Family caregivers (CG) play a vital role in providing care for a person living with dementia $(\mathrm{PwD})$ in the home setting. ${ }^{1}$ This personalised care directly benefits the PwD by keeping them at home in a familiar environment and represents a significant economic contribution to society. CGs often find caregiving satisfying and rewarding, however the task of managing the challenging behavioural and psychological symptoms of dementia (BPSD) can override their capacity to cope, leading to negative physical and mental health including fatigue, depression, burnout and illness. ${ }^{23} \mathrm{~A}$ deterioration in CG wellbeing may have a negative flow-on effect on 
the well-being of the PwD. A negative spiral may develop until the CG can no longer sustain the caring role, and the PwD moves into residential care earlier than desired.

The 2015 global cost of dementia was estimated to be US $\$ 818$ billion, and this figure will continue to increase as the number of people with dementia rises. ${ }^{1}$ Nearly $85 \%$ of costs are related to family and social care, rather than medical care. With this increase and the escalating costs of care, it is time-critical that CG-directed home-based interventions are developed and tested. The 2017 Lancet Commission on Dementia ${ }^{1}$ suggests that pharmacological treatment of BPSD should be restricted to those with very severe symptoms and highlights music therapy as a nonpharmacological intervention that reduces BPSD (p.30).

Systematic reviews indicate that the majority of CG-directed interventions adopted cognitive-behavioural or psychoeducational approaches to address CG coping, depression and BPSD management. ${ }^{45}$ Adherence to programmes was poor because CGs could not commit to the regular programme attendance requirements. ${ }^{45}$ Drawing on social exchange theory, apathy and other BPSD lead to diminished reciprocity between CG and $\mathrm{PwD}$, creating imbalances in the relationship. ${ }^{6}$ Therefore, the convenience of a home-based CG-delivered programme that can manage BPSD and address relationship reciprocity is more likely to be adhered to, and more effective in promoting both $\mathrm{PwD}$ and CG well-being.

Music therapy is a registered psychosocial National Health Service (NHS in the UK) intervention that meets the current recommendations for addressing the individual needs of those with dementia. ${ }^{7}$ HOMESIDE (HOME-based caregiver-delivered music intervention for people living with dementia) uses a purposefully developed music intervention (MI) (described later) informed by previous meta-analyses that demonstrate the effectiveness of music therapy in reducing BPSD. ${ }^{8-11}$ The MI is a translation of the research evidence to a homecare context and, instead of being delivered directly by qualified music therapists, they will train CGs to deliver the MI. The MI incorporates Kitwood's model of personhood for $\mathrm{PwD},{ }^{12}$ which is essential to effective dementia care and underpins the philosophy of the 2018 Alzheimer's Association Dementia Care Practice Recommendations. The person-centred dementia care embedded in the MI emphasises communication and relationships, recognising that dementia is best understood as an interplay between neurological impairment and psychosocial factors (eg, health, individual psychology) and the environment. ${ }^{13}$
Small-scale studies that have tested the effectiveness of MI training programme for informal and formal CGs have had positive findings to date. Results of a cluster randomised controlled trial (RCT) with formal CGs showed the MI to be a practicable and acceptable intervention, with $\mathrm{PwD}$ showing treatment-related improvements, and staff reporting enhanced skills in caregiving. ${ }^{8}$ Although based on a small sample $(n=17)$, large effects in BPSD (Cohen's $\mathrm{d}=2.32$ ) were found between standard care (SC) and MI from baseline to 7 months. A homebased feasibility RCT determined acceptability of the MI, assessed burden associated with delivering the MI and tested appropriateness of the measures. ${ }^{14}$ BPSD scores decreased from baseline to post-test in the MI group but increased in the SC group and mixed results were shown for the comparative reading group. A study involving eight family CGs who were trained to deliver home-based music programmes for their care recipient with dementia found that both CGs and PwD improved in self-reported relaxation, comfort and happiness from baseline to post-test. Music activities taught to CGs comprised music listening with reminiscence, movement to music, music and progressive muscle relaxation, drawing and discussing drawing to music, singing, percussion instrument playing and strategic use of music for use while performing activities of daily living. CGs seemed to derive great benefit from the programme in comparison with care recipients. Findings suggested that CGs enjoyed partaking in the reminiscing and shared musical activities with their loved ones. $^{15}$

The conceptual framework underpinning the MI incorporates the responses of the $\mathrm{PwD}$, the ensuing momentto-moment interaction between the $\mathrm{PwD}$ and the CG and the CGs' responses to the PwD and moments of interaction (figure 1). The MI is grounded in the established knowledge that music-induced emotions and memories are often retained in $\mathrm{PwD}$ because of the relative preservation of medial frontal and limbic areas. ${ }^{9}{ }^{16}$ MIs are effective when the music selected for use is chosen by the PwD (or CG).${ }^{911}$ When music facilitates moment-to-moment interactions, emotional and social engagement and autobiographical recall, imbalances in reciprocity are diminished. CGs' positive experience of seeing 'the person behind the dementia' via this music-induced response evokes CG experiences of pleasure, feelings of competence in the CG and fosters their resilience and coping. Ultimately, the enhanced well-being of CGs will lead to more effective care and better well-being outcomes for both CGs and PwD (figure 1).

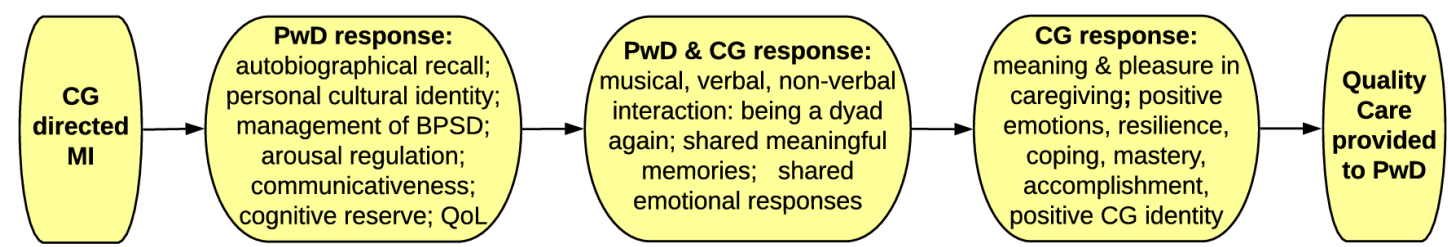

Figure 1 Mechanisms of action underpinning the music intervention. BPSD, behavioural and psychological symptoms of dementia; CG, caregiver; MI, music intervention; PwD, people with dementia; QoL, quality of life. 


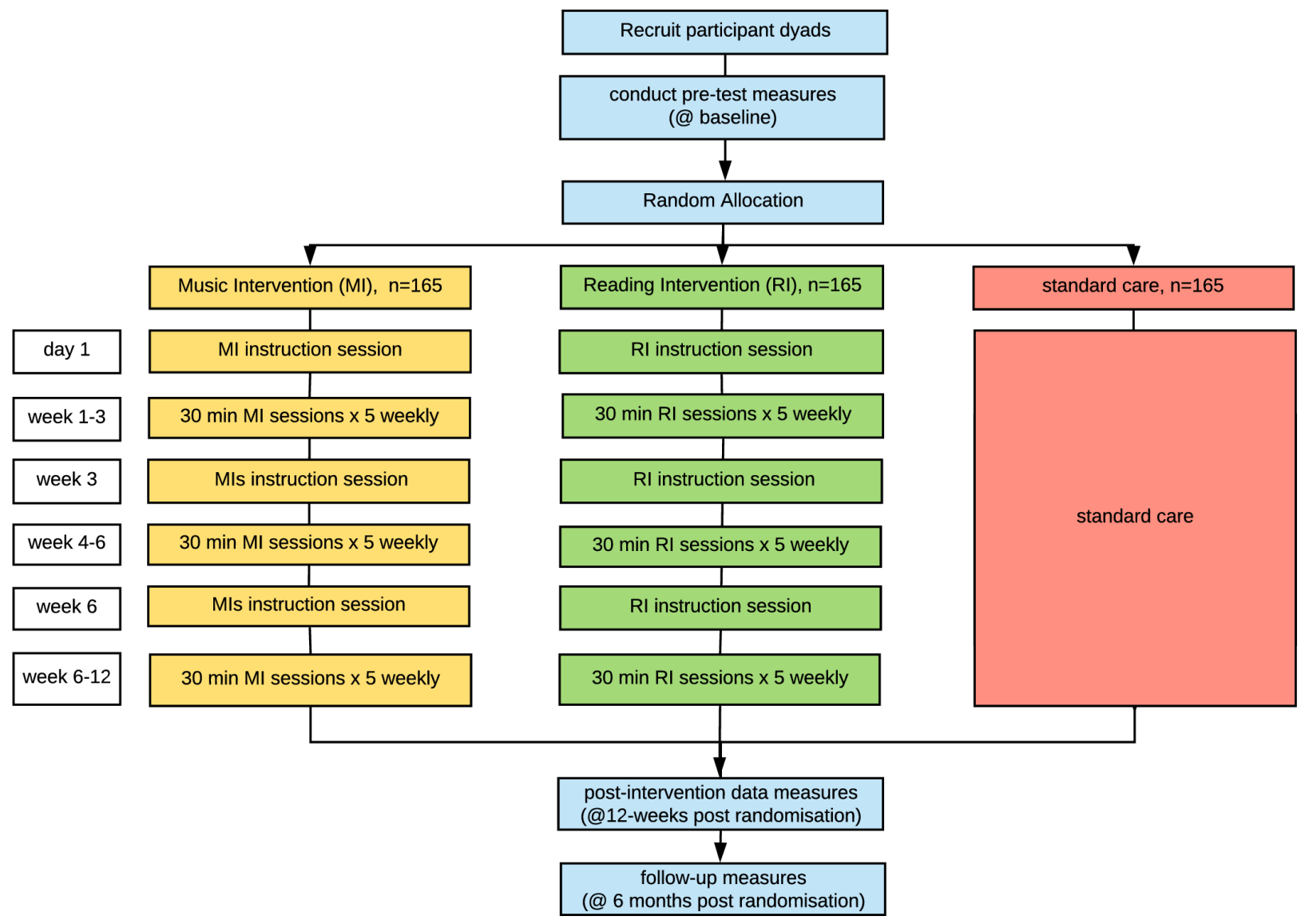

Figure 2 HOMESIDE illustration of study design.

The comparative conditions in this trial are SC only (control) and a reading intervention plus SC (RI, active control). The RI was included because studies have shown preliminary evidence that reading to and with $\mathrm{PwD}$ can have positive impacts on BPSD. ${ }^{17} 18$ We anticipate the MI to be superior to SC only. In addition, we postulate that the RI will lead to a small positive effect but the MI is expected to be superior as music has been shown to be a social connector and a trigger of autobiographical recall, is non-reliant on intact verbal comprehension or expression and can be used to regulate emotion and behaviour. ${ }^{79-11 ~ 16 ~ 19-22 ~}$

\section{Trial design}

A large, pragmatic, single-blinded, international three-arm parallel-group RCT design is planned with a 1:1:1 allocation ratio. Cohabiting dyads where one member of each dyad has a diagnosis of dementia will be randomised to one of three conditions: 1) MI plus SC; 2) RI plus SC and 3) SC only (figure 2). CGs in MI and RI groups will receive a 2-hour training session on how to deliver the MI or RI and will then engage the PwD in a 5x weekly CG-directed home-programme for 12 weeks. Two additional training sessions will be provided at 3 and 6 weeks post allocation. Fifteen-minute fortnightly phone calls will be scheduled to support the CG, and encourage adherence to the protocol. Data will be collected at baseline, at the end of the 12-week intervention and at 3-month follow-up (6 months postrandomisation). The SC group will not receive any training sessions. This trial is framed as a superiority trial where we hypothesise that the MI will be superior to SC (primary) and RI plus SC (secondary) regarding BPSD of PwD at 12 weeks postrandomisation.

\section{Objectives}

The aim is to demonstrate the effectiveness of the 12-week HOMESIDE MI plus SC on the short-term BPSD at the end of intervention of PwD living at home and being cared for by a cohabiting CG compared with SC (primary), and to evaluate the effectiveness of MI plus SC compared with RI plus SC (secondary). Other secondary objectives are as follows:

- Evaluate the maintenance of the effect of the MI plus SC on long-term (6 months postrandomisation) BPSD compared with SC and RI plus SC.

- Evaluate the effectiveness of the MI plus SC on shortterm and long-term levels of depression and QoL of PwD compared with SC and RI plus SC.

- Evaluate the effectiveness of the MI plus SC on shortterm and long-term levels of depression, resilience, sense of competence and QoL of the CG compared with SC and RI plus SC.

- Evaluate the effectiveness of the MI plus SC on the short-term and long-term perceived quality of the relationship between $\mathrm{PwD}$ and CG compared with SC and RI plus SC. 
- Compare the cost-effectiveness of a CG-delivered MI plus SC on PwD and CG outcomes compared with SC and RI plus SC, using health-related QoL for both $\mathrm{PwD}$ and CG.

\section{METHODS AND ANALYSIS \\ Participants}

The trial will be conducted in people's homes located in metropolitan cities and adjoining rural areas in Australia, Germany, Poland, Norway and the UK.

Inclusion criteria:

- Dyads (cohabiting) who are close in relationship and where one member has a diagnosis of dementia according to the 10th revision of the International Statistical Classification of Diseases and Related Health Problems (ICD-10) criteria (Alzheimer's disease $(\mathrm{AD})$, frontotemporal dementia, vascular dementia), Lewy body disease or mixed dementia) as determined by a clinician experienced in diagnosing dementia. ${ }^{7}$ Close in relationship refers to a CG who may be a sibling, spouse, adult child, friend, niece or nephew or any person who has a close relationship to the PwD, that is, anyone who is not a formal paid caregiver.

- Dyads where the PwD has a Neuropsychiatric Inventory-Questionnaire (NPI-Q) score of $\geq 6$ (from a maximum score of 36) and Mini Mental State Examination (MMSE) scores $<24$ as research indicates that NPI-Q scores $>6$ occur in PwD who have high MMSE scores. ${ }^{23}$ The NPI-Q will form part of the screening process, with a trained assessor administering the NPI-Q in the dyad's home prior to enrolment in the study.

Exclusion criteria:

- Dyads where either or both the CG or PwD have significant hearing impairments that are not resolved through the use of a hearing aid device and limit their capacity to enjoy musical experiences. There will be no further exclusions.

\section{Interventions}

\section{Music intervention}

Dyads randomly allocated to the MI will receive a 2-hour home-based MI training session that aims to engage the PwD during and following the MI. Using a carefully prepared and detailed intervention manual, a qualified music therapist will instruct the CG on methods and strategies for using music to assist the PwD to become calmer (if agitated) or more energised (if apathetic). CGs will be instructed on how to choose music and engage the $\mathrm{PwD}$ in effective and respectful discussions with the aim of evoking autobiographical memories and sharing meaningful experiences. ${ }^{24}$ Strategies to engage the PwD and create opportunities for meaningful dialogue with the $\mathrm{PwD}$ will be provided, as well as training CGs to notice the PwD's positive and negative responses to music. The activities to be taught comprise: a) singing familiar/preferred songs followed by CG-facilitated discussions about the meaning of the songs for the dyad, the PwD and significant others, and any associated memories ${ }^{2025}$; b) movement to music (eg, upper body and arms imitating familiar dance movements to music) to assist in regulating arousal ${ }^{11}$; c) playing instruments (or using household items to make rhythmic sounds) while listening to music and d) listening to familiar/preferred relaxing or enlivening music dependent on BPSD present at the time to assist in regulating arousal. ${ }^{20}$ CGs are then instructed to deliver the MI at least 5x per week for approximately 30 min over a 12-week period. After each MI session, they will diarise their experiences, including documenting which activities were used, session time and duration and any positive or negative responses during and after the session. Such data (number of times per week, average duration, activities adopted) will be used to monitor and improve adherence to the protocol. At 3 and 6 weeks post allocation, the MI trainer (music therapist) will return to the dyad's home for a second and third training session (figure 2). These sessions aim to further extend CG knowledge and skills, troubleshoot any issues and improve protocol adherence. Fortnightly phone conversations with CGs will be used to support the CG and remind them to complete the diaries (to mitigate risk of non-completion and maximise participant engagement, retention and protocol adherence).

\section{Reading intervention (active control)}

Dyads randomly allocated to the RI group will receive a 2-hour RI instruction session which aims to engage the PwD during and following the RI. The reading activities will be taught by a qualified practitioner, following a carefully prepared and detailed intervention manual. These activities are based on RI methods commonly used with PwD including: a) CG reading aloud to the PwD; b) PwD reading aloud (or reciting poems, prayers, prose, short stories, fairy tales, when unable to read) to CG; c) listening to audio books and d) discussion of the text and personal responses. ${ }^{17-19}$ Strategies to engage the PwD and create opportunities for meaningful dialogue with the $\mathrm{PwD}$ will be provided as well as guidance on selecting reading material accessible to the PwD's level of cognitive impairment. CGs are then instructed to deliver RI at least 5x per week for 30 min over 12 weeks and diarise their reading activities to record activity and adherence. Diaries will serve as a mechanism to monitor adherence to the protocol. At 3 and 6 weeks post allocation, as per the MI condition, the RI trainers will return to the dyad's home for a second and third training session with the intention of further extending CG knowledge and skills and to monitor and improve intervention protocol adherence. Fortnightly phone conversations with CGs will be used to support the CG and remind them to complete the diaries (to mitigate risk of non-completion). Like the MI condition, phone calls also aim to maximise participant engagement, retention and protocol adherence.

For both the MI and RI, at screening, the assessors will determine the music and reading resources already available to the dyads. Should they require resources (eg, 
large print books, mp3 players/speakers, downloadable music), the research team will loan these resources for the dyads, free of charge.

\section{Standard care}

For dyads randomly allocated to the SC condition, the CG will not be trained in either MI or RI but will be instructed to care for the $\mathrm{PwD}$ in their usual manner. This consists of providing medical, therapeutic and personal care, as well as participating in usual leisure activities.

\section{Discontinuing or modifying Interventions}

Where there is a significant deterioration in the health of the PwD and/or the CG that leads to hospital admission or care home admission, the MI or RI will be discontinued. If there is a change in primary CG partway through the study, the dyad will be withdrawn from the study.

\section{Training and assessment of fidelity}

Given the MI and RI will be delivered in five different countries with different healthcare philosophies and practices, a careful plan for fidelity of the study design, treatment integrity, treatment differentiation, treatment receipt and treatment enactment has been developed. A standardised manual for MI and RI has been developed and agreed on by all countries prior to implementation. Fidelity in this study is complex as it will involve assessing fidelity of the MI and RI training session, and fidelity of the CG-directed programme. Delivery of MI and RI training by research staff will be video-recorded and a randomised selection of $20 \%$ of recordings from every site will be reviewed by members of the research team and cross-checked with the MI and RI protocol manuals using a custom fidelity checklist. Individualised supervision and monitoring of intervention trainers will be employed to minimise 'drift' in trainer differences and control for differences in trainer styles. CG diaries will be used to determine whether the MI and RI protocols have been adhered to and the success of treatment enactment.

\section{Outcomes}

At baseline, demographic data (age, gender and dyad history) of both CG and PwD will be collected as well as diagnostic information of PwD (ICD-10).

Where possible, core outcomes (www.comet-initiative. org) for psychosocial intervention research in dementia care were selected. ${ }^{26}$ For the $\mathrm{PwD}$, the following measures were selected:

- BPSD: the NPI-Q is the most highly regarded and used measure for determining the severity of BPSD in clinical trials. The 12-item scale is used to assess the behaviour of PwD across 12 domains of commonly displayed BPSD. The scale has been translated into $>40$ languages, has been cross-validated against the NPI as the gold standard $(\mathrm{r}=0.73)$ and has demonstrated good validity $($ sensitivity $=74.1 \%$, specificity $=79.5 \%)$, internal reliability $(\alpha=0.783)$ and excellent test-retest reliability $(\mathrm{r}=0.99) .{ }^{27} 28$ Total severity scores range from 0 to 36 ; higher values are indicative of higher severity. Distress scores range from 0 to 60 ; higher values represent higher levels of distress. CGs will selfcomplete the NPI-Q with guidance from the research assessor if required.

- Depression: the Montgomery Asberg Depression Rating Scale (MADRS) ${ }^{29}$ will be used to assess the severity of depression. Scores for each of the 10 items range from 0 (no symptoms) to 6 (severe symptoms) and are determined through an assessor-led interview with the proxy, in this study, the CG. Total score ranges from 0 to 60 with higher scores indicating more severe depression. The MADRS has been found to have good constructive validity, internal reliability $(\alpha=0.84)$ and test-retest reliability (intraclass correlation coefficient $=0.78$ ). The scale has been widely used in clinical trials. ${ }^{29}$

- QoL: the QoL of PwD will be determined by administering the QoL-AD ${ }^{30}$ scale. The QoL-AD is recommended by the COMET Initiatve, ${ }^{26}$ for use in clinical effectiveness trials. It is a simple 13-item self-report measure, which is rated on a 4-point scale, within the structure of a verbally delivered interview. Total scores range from 13 (poor QoL) to 52 (excellent QoL in all areas). Studies indicate that the measure can demonstrate sensitivity to psychosocial intervention, correlates with health-utility measures,${ }^{31}$ has excellent inter-rater reliability $(\kappa>0.70)$ and internal consistency $(\alpha=0.82)$. The QoL-AD is reliable when used with people with MMSE scores of $>10$. Both a CG proxy and PwD self-report (if possible) will be collected at the three time points. If the $\mathrm{PwD}$ is able to complete the MMSE at all time points, then their response will be included in the analysis. If not, then the proxy version at all time points will be used.

- Cognition: the MMSE will be administered preintervention and postintervention (time 1 and time 2) to monitor any change in the PwD's cognition and to examine the relationship between cognitive decline, BPSD, depression and response to different conditions. The MMSE is a 30-point questionnaire used to estimate the severity and progression of cognitive impairment and to follow the course of cognitive changes in an individual over time. ${ }^{32}$ The MMSE tests for orientation, attention, memory, language and visual-spatial skills. It is reliable and valid for both diagnosis and longitudinal assessment. Higher scores indicate better cognitive capacity with scores of 24-30 indicating no cognitive impairment; 19-23 indicating mild cognitive impairment; 10-18 indicating moderate cognitive impairment and scores $<10$ indicating severe cognitive impairment. MMSE scores will be determined through assessor-led interviews with PwD participants.

For the CG, the following measures were selected:

- Depression: CG depression will be measured using the Patient Health Questionnaire-9 (PHQ-9). ${ }^{33}$ This self-completed 9-item questionnaire asks the participant about how often they experience the descriptors 
over the last 2 weeks. Each item is scored from 0 (not at all) to 3 (nearly every day). Total scores range from 0 to 27. The PHQ-9 has comparable sensitivity and specificity to other depression measures; high internal reliability $(\alpha=0.89)$ and test-retest reliability $(r=0.84)$.

- Resilience: CG resilience will be measured using the self-completed 14-item Resilience Scale. ${ }^{34}$ Total scores range from 14 to 98 , with higher scores indicative of higher resilience. The measure has been tested and has good concurrent validity, good internal reliability $(\alpha=0.8-0.90)$, good construct validity, test-retest reliability $(\mathrm{r}=0.67-0.84)$ and has been translated into 36 languages. ${ }^{35}$

- Competence: CG competence will be measured using the self-completed Short Sense of Competence Questionnaire. ${ }^{36}$ The 7 -items cover three main domains: self-reported feelings about how the caregiver role impacts the CG's personal life, satisfaction with their performance as a CG and their satisfaction with how the PwD responds to the CG. Total scores ranged from 7 to 35 with higher scores indicative of a stronger sense of competence. The measure has been crossvalidated with the longer 35-item standard Sense of Competence Questionnaire $(\mathrm{r}=0.88)$ and has been shown to have high reliability (Cronbach's $\alpha=0.76$ ).

- QoL: CG QoL will be assessed using the self-completed Assessment of Quality of Life-6D instrument. ${ }^{37}$ Each item asks participants to describe their situation over the past week by ticking the box (from 4 to 6 choices) that best reflects their situation. The psychometric property testing found the instrument to be reliable and valid, and has greater sensitivity to the psychosocial dimensions of QoL than other utility instruments. ${ }^{3738}$

- Relationship quality: CG perception of quality of the CG and PwD relationship will be captured by asking the CG to self-complete the Quality of CaregiverPatient Relationship. ${ }^{39}$ This 14 -item measure aims to capture the strength of the quality of relationship between the PwD and CG, from the CG's perspective. Total scores range from 14 to 70 , with higher scores indicating a higher quality relationship. The measure has demonstrated acceptable internal consistency $(\alpha=0.82)$ and concurrent validity.

- Adherence to the MI/RI intervention will be measured through CG completed diaries. CGs are deemed adherent to the protocol if they have provided $>2$ sessions of MI or RI per week, for at least $30 \mathrm{~min}$ in total. Data on the general use of reading and music by all dyads (including SC) will be collected at post-test. For each diary entry, CGs will be asked to record the date, start and stop time of MI/RI engagement, types of activities used, their experiences during the session (negative, neutral, positive, unsure), effects from the intervention for the remainder of the day until the PwD goes to bed for the evening sleep (negative, neutral, positive, unsure) and any comments. These data will be used in qualitative analyses to gain more nuanced understandings of how the activities are perceived and how these may change over the course of the intervention period.

To evaluate the cost-effectiveness of MI compared with RI and SC on PwD and CG, the following outcomes will be measured:

- Quality-adjusted life-years (QALYs): the EuroQol instrument (EQ-5D-5L) ${ }^{40}$ is a generic QoL measure that is internationally used to determine the QALYs and used for clinical and economic appraisal. ${ }^{40}$ The measure is quick to complete and not cognitively demanding. Scores from the five items are not combined arithmetically but using preference weights which arrive at an overall QoL score. These range from lower than 0 (worse than death) to 1 (best possible). PwD will complete the self-report version of the EQ-5D-5L. CGs will complete the measure as a proxy for the $\mathrm{PwD}$ and self-report their own health status. ${ }^{26} 41-44$

- Resource utilisation in dementia (RUD): the RUD is a standardised instrument for resource use data collection in dementia, designed to collect data from formal and informal care across different countries. The RUD assesses resource use of both PwD and CG, including time expended in different daily tasks, and consists of baseline and a follow-up assessment. ${ }^{45}$ The RUD will be completed through assessor-led interviews with CGs.

CGs will be provided with a set of guidelines as to how to complete the diary and all self-report and proxy measures. The schedule for enrolment, baseline assessments, all outcome measures, and intervention trainings is outlined in figure 3.

\section{Sample size}

A total of 165 dyads in each arm of the study, or 495 in total, are needed to detect a difference of 3-points in NPI-Q total severity score (primary outcome) between the MI plus SC and SC arm (primary comparison) at 12 weeks (primary time point). This assumes $90 \%$ power, a two-tailed significance level of $5 \%$, equal SD (7.5 points) in the groups, no correlation between baseline and 12 weeks (conservative) and 20\% attrition. A 3-point change from baseline in NPI-Q total severity score is considered a clinically meaningful difference. ${ }^{46}$ A conservative SD of 7.5 points is based on that observed in 1026 communityliving participants across eight European countries with mild-to-severe dementia (SD 5.9-6.5 points) ${ }^{47} 48$ A conservative drop-out proportion (eg, withdrawn by CG, physician or death) of $20 \%$ is based on a reported $5.6 \%(95 \%$ CI $1.8 \%$ to $12.6 \%$ ) drop-out at 3 months in 89 inpatients with mild-to-moderate dementia in Finland participating in a three-arm RCT of singing, music or usual care, ${ }^{25}$

\section{Recruitment}

Randomisation will aim to be distributed equally across five countries (Australia, the UK, Norway, Germany and Poland) to support between-country analyses. Participants 


\begin{tabular}{|c|c|c|c|c|c|c|c|}
\hline \multirow[b]{2}{*}{ TIMEPOINT } & \multirow{2}{*}{$\begin{array}{c}\text { Enrolment } \\
\text { Day }-1 \\
(-7--1 \\
\text { days })\end{array}$} & \multirow{2}{*}{$\begin{array}{c}\text { Allocation } \\
0\end{array}$} & \multicolumn{3}{|c|}{ Intervention } & \multirow{2}{*}{$\begin{array}{c}\begin{array}{c}\text { Post- } \\
\text { Intervention }\end{array} \\
\begin{array}{c}\text { Day } 90 \\
(+/-7 \text { days })\end{array} \\
\end{array}$} & \multirow{2}{*}{$\begin{array}{l}\text { Follow- } \\
\text { up } \\
\text { Day } 180 \\
(+/-7 \\
\text { days }) \\
\end{array}$} \\
\hline & & & $\begin{array}{l}\text { Day } 1 \\
(+/-7 \\
\text { days }) \\
\end{array}$ & $\begin{array}{c}\text { Day } 21 \\
(+/-7 \\
\text { days }) \\
\end{array}$ & $\begin{array}{c}\text { Day } 42 \\
(+/-7 \\
\text { days }) \\
\end{array}$ & & \\
\hline \multicolumn{8}{|l|}{ ENROLMENT: } \\
\hline \multirow{3}{*}{$\begin{array}{r}\text { Informed consent (or assent) } \\
\text { Dyad: allocation }\end{array}$} & $\mathrm{x}$ & & & & & & \\
\hline & $x$ & & & & & & \\
\hline & & $\mathrm{x}$ & & & & & \\
\hline \multirow{3}{*}{$\begin{array}{r}\text { INTERVENTIONS: } \\
\text { Music Intervention Training } \\
\text { Reading Intervention Training }\end{array}$} & & & & & & & \\
\hline & & & $\mathrm{x}$ & $x$ & $x$ & & \\
\hline & & & $\mathrm{x}$ & $\mathrm{x}$ & $\mathrm{x}$ & & \\
\hline \multicolumn{8}{|l|}{ ASSESSMENTS: } \\
\hline $\begin{array}{l}\text { PERSON WITH DEMENTIA } \\
\text { sociodemographic information }\end{array}$ & $x$ & & & & & & \\
\hline \multirow{2}{*}{$\begin{array}{r}\text { MMSE (self) } \\
\text { dementia diagnosis (proxy) }\end{array}$} & $\mathrm{x}$ & & & & & $\mathrm{x}$ & \\
\hline & $\mathrm{x}$ & & & & & & \\
\hline NPI-Q (proxy) & $\mathrm{x}$ & & & & & $\mathrm{x}$ & $\mathrm{x}$ \\
\hline MADRS (proxy) & $\mathrm{x}$ & & & & & $\mathrm{x}$ & $\mathrm{x}$ \\
\hline \multirow{3}{*}{$\begin{array}{r}\text { QoL-AD (self and proxy) } \\
\text { EQ-5D-5L (self and proxy) } \\
\text { Adverse events; death, } \\
\text { hospitalisation, death of CG }\end{array}$} & $\mathrm{x}$ & & & & & $\mathrm{x}$ & $\mathrm{x}$ \\
\hline & $\mathrm{x}$ & & & & & $\mathrm{x}$ & $\mathrm{x}$ \\
\hline & & & $x$ & $x$ & $x$ & $\mathrm{x}$ & $x$ \\
\hline $\begin{array}{r}\text { CAREGIVER } \\
\text { sociodemographic information }\end{array}$ & $x$ & & & & & & \\
\hline PHQ-9 & $\mathrm{x}$ & & & & & $\mathrm{x}$ & $x$ \\
\hline RS-14 & $x$ & & & & & $\mathrm{x}$ & $\mathrm{x}$ \\
\hline SSCQ & $\mathrm{x}$ & & & & & $\mathrm{x}$ & $\mathrm{x}$ \\
\hline QPCR & $\mathrm{x}$ & & & & & $\mathrm{x}$ & $\mathrm{x}$ \\
\hline AQoL-6D & $\mathrm{x}$ & & & & & $\mathrm{x}$ & $\mathrm{x}$ \\
\hline EQ-5D-5L(self) & $\mathrm{x}$ & & & & & $\mathrm{x}$ & $\mathrm{x}$ \\
\hline RUD & $\mathrm{x}$ & & & & & $\mathrm{x}$ & $\mathrm{x}$ \\
\hline Post-training Questionnaires & & & $\mathrm{x}$ & $\mathrm{x}$ & $\mathrm{x}$ & & \\
\hline Interviews & & & & & & $\mathrm{x}$ & \\
\hline $\begin{array}{r}\text { Diary (5 x weekly diary entries } \\
\text { for MI and RI) }\end{array}$ & & & $x$ & $x$ & $x$ & $x$ & \\
\hline $\begin{array}{r}\text { Adverse events; death, } \\
\text { hospitalisation }\end{array}$ & & & $x$ & $x$ & $x$ & $x$ & $x$ \\
\hline
\end{tabular}

Figure 3 Schedule of enrolment, interventions and assessments. AQoL-6D, assessment of quality of life-6D instrument; EQ5D-5L, the EuroQol instrument; ICD-10, 10th revision of the International Statistical Classification of Diseases and Related Health Problems; MADRS, Montgomery Asberg Depression Rating Scale; MI, music intervention; MMSE, Mini Mental State Examination Score; NPI-Q, Neuropsychiatric Inventory; PHQ-9, Patient Health Questionnaire-9; QALY, quality-adjusted lifeyears; QCPR, quality of caregiver-patient relationship; QoL-AD, quality of life-Alzheimer's disease; RI, reading intervention; RS, Resilience Scale; RUD, resource utilisation in dementia; SSCQ, Short Sense of Competence Questionnaire.

will be recruited through established partner organisations. Staff from the partner organisations will introduce the trial to potential participants and invite them to participate in the study. They will be given an information sheet explaining the main aspects of the trial and provided with contact details of the research team who will be available to answer further questions.

\section{Randomisation, allocation concealment and blinding}

The randomisation schedule will be computer-generated by an independent statistician and allocation will be carried out through a centralised randomisation service. Block permuted randomisation with stratification by participating site will be used, so that treatment balance within site is achieved. Dyads who meet the inclusion criteria and none of the exclusion criteria will be randomised 1:1:1 into MI, RI or SC. Randomisation will occur after the eligibility checking, informed consent and baseline assessment have been completed. Informed consent/assent will be obtained by a blinded assessor prior to the baseline assessment. The study coordinator in each country will be informed of the allocation and will inform dyads of their group allocation by post, phone or email. 
Participant dyads cannot be fully blinded due to the active nature of the interventions; however, plain language statements and consent forms will use neutral wording to maintain equipoise and to avoid expectancy effects. Blinded assessors will collect participants' data at baseline, postintervention and follow-up. Diaries will be returned in sealed envelopes to minimise risk of assessors becoming unblinded. The success of assessor blinding will be checked by asking the assessor to guess the treatment assignment (or say "I do not know") after the postintervention and follow-up periods. This treatment guess will then be compared against the actual treatment and the blinding index derived. The independent statistician will not reveal the allocation codes to any of the study team except for the study coordinators of each country in charge of group allocation. All other investigators and the study statistician will remain blinded until the database has been cleaned, a blinded data review has taken place and the database is ready for analysis.

\section{Analysis}

Analyses will be performed on an intention-to-treat basis including all randomised dyads in their allocated study arm. The primary outcome (NPI-Q severity total score) will be analysed using a constrained longitudinal data analysis (cLDA) model, ${ }^{49}$ with response consisting of all scores (baseline, 12 weeks and 6 months) and the model including factors representing intervention, time, intervention by time interaction and site with the restriction of a common baseline mean score across interventions. This refers to the assumption that at baseline there are no differences between the interventions in the mean score, thus assuming the randomisation was effective. This assumption will be enforced statistically in the statistical model. The absolute difference between MI and SC and MI and RI in mean change from baseline will be estimated (including two-sided 95\% CI) at 12 weeks (primary time point). A hierarchical fixed sequence testing procedure will allow testing of MI versus RI at 12 weeks at $5 \%$ if the comparison of MI versus SC at 12 weeks has a $p$ value $<0.05$. Secondary analyses will consist of a model adjusted for potential confounders (types of dementia and gender). The cLDA model provides valid inference if the missing data mechanism is at most missing at random. In addition to the intention-to-treat effect, we will obtain the complier average causal effect by making use of the collected adherence data. ${ }^{50}$ Analyses similar to the primary outcome will be applied to the secondary outcomes for PwD and CGs. Heterogeneity of the intervention effect across subgroups (gender of the $\mathrm{PwD} / \mathrm{CG}$, types of dementia, severity of dementia, time of onset dementia, length of time having dementia, relationship between PwD and CG, country) will be assessed by means of interaction tests. The number and percentage of PwDs and CGs with adverse events will be summarised by intervention group.

Cost-effectiveness analysis in a societal perspective will be performed separately for each country using the utilities generated by EQ-5D-5L for both PwD and CG and country-specific weights, to estimate a combined QALY score using a generalised linear model adjusted by the baseline. Health and informal care resources consumed by $\mathrm{PwD}$ will be assessed using the RUD and unit cost by country. A generalised linear mixed model will be used to estimate the main predictors of the total costs in the MI, RI and SC groups. Incremental cost-effectiveness ratio will be calculated using the cost and effect estimates comparing MI with RI and SC. The uncertainty around the incremental cost-effectiveness ratio will be estimated using bootstrapping (1000 replications) adjusting to control variables.

\section{Patient and public involvement}

All countries have involved user and advocacy organisations in the development and design of the study. The UK, Australia and Germany have been piloting work with formal and informal CGs of PwD for many years. CGs and $\mathrm{PwD}$ have been involved designing the diaries which capture adherence data. It was imperative that the diary be user-friendly, not burdensome on the CG, and yet enabled them to document both the positive and negative aspects of the session. Several iterations of the diary were constructed prior to arriving at the final structure. Pilot work in Australia ${ }^{14}$ involved interviews postintervention to identify strengths, limitations, challenges and experiences in delivering the MI, as well as recommendations for suggested modifications to the intervention training. Representatives of advocacy groups and end users from all countries will be represented on an international Participant and Public Involvement Committee.

\section{Monitoring and oversight}

A Trial Operations Committee (TOC) will comprise the principal investigator, chief investigators and clinical trial managers from each of the five countries, the study statistician, health economist and a consumer representative. The TOC will meet at least 6 -weekly, and will oversee all aspects of the trial delivery including strategies to support efficient and effective recruitment and retention, reviewing completeness of data sets, monitoring intervention fidelity, management of timelines and milestones, review of country-by-country progress, public and patient involvement or actions and publication and dissemination plans. The role of the members of the TOC is to bring country-specific issues to the international team for discussion to ensure the study is being monitored and delivered according to the agreed protocols. Protocol deviations and any changes or amendments to the operational processes of the trial will be discussed and decisions made by the TOC.

A Trial Steering Committee will comprise members independent of the clinical trial including consumer representatives and representatives from other relevant advocacy organisations. The trial principal investigator (or a proxy in her absence) will also sit on the committee as a non-voting member. The Committee will 
meet biannually (or more often when needed) to review and monitor all aspects of the study delivery. They will draw on reports provided to them by the TOC and make recommendations to the principal investigator and TOC about whether further actions are required.

A Data Safety Management Committee (DSMC) will review the cumulative study data to evaluate the recruitment, safety, study conduct and scientific validity and integrity of the trial. The committee consists of at least five people with strong methodological, biostatistical and clinical expertise who are independent of the project and an end user representative. The DSMC will be provided with data on recruitment, intervention uptake, any unforeseen and/or adverse events and review serious adverse events. The meetings will consist of an open and a closed part. In the open part, the general progress of the trial will be discussed with the principal investigator. In the closed part, the DSMC will discuss any safety concerns and if considered required, the DSMC will make recommendations to the TOC for appropriate action.

Study data will be collected and managed using REDCap electronic data capture tools hosted at the University of Melbourne. ${ }^{51}$ The Data Management Coordinating Center will oversee the intrastudy data sharing process between countries, with input from the Data Management Subcommittee. We will develop a data management manual detailing data collection protocols and provide comprehensive training of those members of the research team who collect, check and enter study data. The principal investigator, study statistician and health economist from the University of Melbourne will be given access to the cleaned data sets. Country-specific lead investigators will only have access to their own country's cleaned data sets. All data sets will be password-protected. To ensure confidentiality, data dispersed to project team members will have any identifying information removed.

\section{Risk management}

Processes have been put in place to mitigate risks. One of the most significant risks associated with the project is slow recruitment. To offset the risk of slow recruitment, data are being collected across five countries. If some countries have less difficulty than others in recruiting, then these countries will recruit greater numbers to ensure the required sample size is obtained. Another risk identified is the heterogeneity of intervention delivery. The inclusion of a detailed manual, regular supervision with interventionists, and monitoring the effectiveness of interventionist training will mitigate the risk of poor intervention fidelity.

\section{Ethics and dissemination plan}

All research and clinical activities carried out for the HOMESIDE project will be in compliance with fundamental ethical principles including those reflected in the Oviedo convention and the Convention for the Protection of Human Rights and Fundamental Freedoms and legal requirements (Directive 95/46/EC on the protection of individuals with regard to the processing of personal data and on the free movement of such data; and Directives 2001/20/EC, 2005/28/EC relating to the implementation of good clinical practice in the conduct of clinical trials). Ethical conduct will be managed in the following ways:

- The clinical trial coordinator in each country will implement the research in full respect of European/ national/ institutional legal and ethical requirements and codes of practice.

- Ethics approvals in each country must be obtained prior to commencement of the trial.

- Informed consent from the PwD's guardian must be obtained prior to enrolling a participant in the study. Assent from the $\mathrm{PwD}$ will always be sought prior to enrolment in the study.

- National and international rules on data protection will be followed. Participating countries in HOMESIDE within the EU and EEA (UK, Germany, Poland and Norway) also relate to the General Data Protection Regulation (GDPR)(Regulation (EU) 2016/679), designed to harmonise data privacy laws across Europe, to protect and empower all EU citizens data privacy and to reshape the way organisations across the region approach data privacy. The HOMESIDE partners have also signed a consortium agreement where they consent to follow national and international rules on collaboration, ethics and data protection.

The report on the main, preplanned analyses of the primary end point and up until the 6-month follow-up will be submitted to a leading medical journal. Further publications may focus on issues such as recruitment and retention strategies for home-based programmes. Publications based on qualitative interviews and video analyses will focus on barriers and facilitators for implementation and promotion of adherence to home-based programmes; experiences of caregivers in delivering the programmes and the development of best practice training guidelines. In addition to publications in academic journals, a number of policy briefing papers for government and aged care/dementia advocacy groups are planned as well as the development of training manuals and guidelines for dissemination.

\section{Data sharing}

In accordance with the Australian Code for Responsible Conduct of Research (Universities Australia, 2018), all data will be retained for retrieval and reuse in future research where participant permission is granted. Following project completion, de-identified anonymised data (with participant consent) will be available on the Australian Data Archive https://ada.edu.au and listed on Research Australia's https://researchaustralia. org website to facilitate access for future research. Data made available will include individual-level deidentified participant data, reports on adverse events and deidentified interview transcripts. According to the GDPR, the 
consortium have agreed to the reuse of data for 10 years post project completion.

\section{Relevance and benefit to society}

As the majority of PwD live in the community and not in residential care settings, quality informal care for $\mathrm{PwD}$ is crucial for managing BPSD and enhancing QoL. This protocol details the process for testing the effectiveness and cost-effectiveness of a CG-directed MI and RI designed to manage the BPSD of PwD, the sense of burden and well-being of the CG and provide meaningful possibilities to maintain the relationship between PwD and their CGs. We expect that with support and training, the MI will be easily implemented in the family home by CGs. With the increasing number of people living with dementia and the stress this will place on countries' economies, our project aims to test an intervention designed to keep people living at home with family CGs for as long as possible, reducing the burden for society and caregivers. Our study will be able to estimate the incremental costeffectiveness ratio between MI and RI, MI and SC and RI and SC. Data may support aged care policy recommendations and as the interventions will be delivered in five different countries, results will be broadly generalisable.

\section{Author affiliations}

${ }^{1}$ Faculty of Fine Arts and Music, The University of Melbourne, Melbourne, Victoria, Australia

${ }^{2}$ Cambridge Institute for Music Therapy Research, Anglia Ruskin University, Cambridge, UK

${ }^{3}$ School of Population and Global Health, The University of Melbourne, Melbourne, Victoria, Australia

${ }^{4}$ Department of Occupational Therapy, University of Physical Education, Kraków, Poland

${ }^{5}$ Austin Health, Melbourne, Victoria, Australia

${ }^{6}$ Centre for Research in Music and Health, Norwegian Academy of Music, Oslo, Norway

${ }^{7}$ Department of Psychiatry, The University of Melbourne, Melbourne, Victoria, Australia

${ }^{8}$ NorthWestern Mental Health, Melbourne Health, Melbourne, Victoria, Australia ${ }^{9}$ Hochschule für angewandte Wissenschaften Würzburg-Schweinfurt, Würzburg, Germany

Contributors FAB took the initiative for the study; FAB, HO-M, TW, KAS, AB, JB developed the concept and design; $\mathrm{SB}$ developed the statistical analysis plan and TVS designed the health economics plan. IC, MHH, TK, NL, Y-ECL, AS-R, JT helped to revise the concept and design. FAB drafted the manuscript; all authors revised the manuscript for important intellectual content. All authors approved the final version of the manuscript.

Funding This work was supported by the Joint Programs for Neurodegenerative Diseases (JPND), which includes joint funding from the National Health and Medical Research Council (APP1169867), Norwegian Research Council (Project number 298995), Federal Ministry of Education and Research Germany (01ED1901) and The National Centre for Research and Development, Poland JPND/04/2019-The National Centre for Research and Development and Alzheimer's Society, UK (grant no. 462).

Competing interests None declared.

Patient consent for publication Not required.

Ethics approval Australia Human Ethics Sub-Committee approval no. 1852845, the United Kingdom approval no. 19/EE/0177, Poland approval no. 186/KBL/ OIL/2019, German approval no. DGP 19-013 and Norwegian Centre for Research Data (Ref 502736) and Norwegian REK Medical and Research Ethics (Ref 2019/941).
Provenance and peer review Not commissioned; externally peer reviewed.

Open access This is an open access article distributed in accordance with the Creative Commons Attribution Non Commercial (CC BY-NC 4.0) license, which permits others to distribute, remix, adapt, build upon this work non-commercially, and license their derivative works on different terms, provided the original work is properly cited, appropriate credit is given, any changes made indicated, and the use is non-commercial. See: http://creativecommons.org/licenses/by-nc/4.0/.

ORCID iD

Felicity Anne Baker http://orcid.org/0000-0002-9851-7133

\section{REFERENCES}

1 Livingston G, Sommerlad A, Orgeta V, et al. Dementia prevention, intervention, and care. Lancet 2017;390:2673-734.

2 Papastavrou E, Kalokerinou A, Papacostas SS, et al. Caring for a relative with dementia: family caregiver burden. $J$ Adv Nurs 2007;58:446-57.

3 O'Dwyer S, Moyle W, van Wyk S. Suicidal ideation and resilience in family carers of people with dementia: a pilot qualitative study. Aging Ment Health 2013;17:753-60.

4 Li R, Cooper C, Austin A, et al. Do changes in coping style explain the effectiveness of interventions for psychological morbidity in family carers of people with dementia? A systematic review and meta-analysis. Int. Psychogeriatr. 2013;25:204-14.

5 Piersol CV, Canton K, Connor SE, et al. Effectiveness of interventions for caregivers of people with Alzheimer's disease and related major neurocognitive disorders: A systematic review. Am J Occup Ther 2017;71:7105180020p1-7105180020.

6 de Vugt ME, Stevens F, Aalten P, et al. Behavioural disturbances in dementia patients and quality of the marital relationship. Int $J$ Geriatr Psychiatry 2003;18:149-54.

7 National Institute for Health and Care Excellence. NICE guidelines: dementia: assessment, management and support for people living with dementia and their carers, 2018. Available: nice.org.uk/ guidance/ng97

8 Hsu MH, Flowerdew R, Parker M, et al. Individual music therapy for managing neuropsychiatric symptoms for people with dementia and their carers: a cluster randomised controlled feasibility study. BMC Geriatr 2015;15:84.

9 Fusar-Poli L, Bieleninik Łucja, Brondino N, et al. The effect of music therapy on cognitive functions in patients with dementia: a systematic review and meta-analysis. Aging Ment Health 2018;22:1103-12.

10 Zhang Y, Cai J, An L, et al. Does music therapy enhance behavioral and cognitive function in elderly dementia patients? A systematic review and meta-analysis. Ageing Res Rev 2017;35:1-11.

11 Ridder HMO, Stige B, Qvale LG, et al. Individual music therapy for agitation in dementia: an exploratory randomized controlled trial. Aging Ment Health 2013;17:667-78.

12 Kitwood T. Dementia reconsidered: the person comes first. Buckingham, UK: Open University Press, 1997.

13 Brooker D. Person-Centred dementia care: making services better. London: Jessica Kingsley Publishers, 2007.

14 Baker F, Grocke D, Pachana N. Connecting through music: a study of a spousal carerCG-directed music intervention designed to prolong fulfilling relationships in couples where one person has dementia. Aust J Music Therapy 2012;23:4-19.

15 Hanser SB, Butterfield-Whitcomb J, Kawata M, et al. Home-based music strategies with individuals who have dementia and their family caregivers. J Music Ther 2011;48:2-27.

16 Jacobsen J-H, Stelzer J, Fritz TH, et al. Why musical memory can be preserved in advanced Alzheimer's disease. Brain 2015;138:2438-50.

17 Longden E, Davis P, Carroll J, et al. An evaluation of shared reading groups for adults living with dementia: preliminary findings. J Public Ment Health 2016;15:75-82.

18 Kawashima R, Okita K, Yamazaki R, et al. Reading Aloud and arithmetic calculation improve frontal function of people with dementia. J Gerontol A Biol Sci Med Sci 2005;60:380-4.

19 Vink AC, Birks JS, Bruinsma MS, et al. Music therapy for people with dementia. Cochrane Database Syst Rev 2004;3:CD003477.

20 Ray KD, Mittelman MS. Music therapy: a nonpharmacological approach to the care of agitation and depressive symptoms for nursing home residents with dementia. Dementia 2017;16:689-710.

21 Aalbers S, Fusar-Poli L, Freeman RE, et al. Music therapy for depression. Cochrane Database Syst Rev 2017;20.

22 van der Steen JT, van Soest-Poortvliet MC, van der Wouden $\mathrm{JC}$, et al. Music-based therapeutic interventions for people with dementia. Cochrane Database Syst Rev 2017;27. 
23 Siafarikas N, Selbaek G, Fladby T, et al. Frequency and subgroups of neuropsychiatric symptoms in mild cognitive impairment and different stages of dementia in Alzheimer's disease. Int Psychogeriatr 2018;30:103-13.

24 Baird A, Thompson WF. The impact of music on the self in dementia. $J$ Alzheimers Dis 2018;61:827-41.

25 Särkämö T, Tervaniemi M, Laitinen S, et al. Cognitive, emotional, and social benefits of regular musical activities in early dementia: randomized controlled study. Gerontologist 2014;54:634-50.

26 Moniz-Cook E, Vernooij-Dassen M, Woods R, et al. A European consensus on outcome measures for psychosocial intervention research in dementia care. Aging Ment Health 2008;12:14-29.

27 Kaufer DI, Cummings JL, Ketchel P, et al. Validation of the NPI-Q, a brief clinical form of the neuropsychiatric inventory. $J$ Neuropsychiatry Clin Neurosci 2000;12:233-9.

28 Wong A, Cheng S-T, Lo ESK, et al. Validity and reliability of the neuropsychiatric inventory questionnaire version in patients with stroke or transient ischemic attack having cognitive impairment. $J$ Geriatr Psychiatry Neurol 2014;27:247-52.

29 Fantino B, Moore N. The self-reported Montgomery-Åsberg depression rating scale is a useful evaluative tool in major depressive disorder. BMC Psychiatry 2009;9:26.

30 Logsdon RG, Gibbons LE, McCurry SM, et al. Assessing quality of life in older adults with cognitive impairment. Psychosom Med 2002;64:510-9.

31 Thorgrimsen L, Selwood A, Spector A, et al. Whose quality of life is it anyway: The validity and reliability of the Quality of Life-Alzheimer's Disease scale. Alzheimer Dis Assoc Disor 2003;17:201-8.

32 Pangman VC, Sloan J, Guse L. An examination of psychometric properties of the Mini-Mental state examination and the standardized Mini-Mental state examination: implications for clinical practice. App/ Nurs Res 2000;13:209-13.

33 Kroenke K, Spitzer RL, Williams JB, et al. The PHQ-9: validity of a brief depression severity measure. J Gen Intern Med 2001;16:606-13

34 Cosco TD, Kaushal A, Richards M, et al. Resilience measurement in later life: a systematic review and psychometric analysis. Health Qual Life Outcomes 2016;14.

35 Wagnild GM, Young HM. Development and psychometric evaluation of the resilience scale. J Nurs Meas 1993;1:165-78.

36 Vernooij-Dassen MJFJ, Felling AJA, Brummelkamp E, et al. Assessment of caregiver's competence in dealing with the burden of caregiving for a dementia patient: a Short Sense of Competence Questionnaire (SSCQ) suitable for clinical practice. J Am Geriatr Soc 1999;47:256-7.
37 Richardson JRJ, Peacock SJ, Hawthorne G, et al. Construction of the descriptive system for the assessment of quality of life AQoL-6D utility instrument. Health Qual Life Outcomes 2012;10:38.

38 Richardson J, lezzi A, Khan MA, et al. Cross-national comparison of twelve quality of life instruments: MIC paper 2 Australia. Victoria, Australia: Monash University, 2012.

39 Spruytte N, Audenhove C, Lammertyn F, et al. The quality of the caregiving relationship in informal care for older adults with dementia and chronic psychiatric patients. Psychol Psychother 2002;75:295-311.

40 Herdman M, Gudex C, Lloyd A, et al. Development and preliminary testing of the new five-level version of EQ-5D (EQ-5D-5L). Qual Life Res 2011;20:1727-36.

41 EuroQol Group. EuroQol - a new facility for the measurement of health-related quality of life. Health Policy 1990;16:199-208.

42 Orgeta V, Edwards RT, Hounsome B, et al. The use of the EQ-5D as a measure of health-related quality of life in people with dementia and their carers. Qual Life Res 2015;24:315-24.

43 Kunz S. Psychometric properties of the EQ-5D in a study of people with mild to moderate dementia. Qual Life Res 2010;19:425-34.

44 Arons AMM, Krabbe PFM, Schölzel-Dorenbos CJM, et al. Quality of life in dementia: a study on proxy bias. BMC Med Res Methodol 2013;13:110.

45 Wimo A, Gustavsson A, Jönsson L, et al. Application of Resource Utilization in Dementia (RUD) instrument in a global setting. Alzheimer's \& Dementia 2013;9:429-35.

46 Mao H-F, Kuo C-A, Huang W-N, et al. Values of the minimal clinically important difference for the neuropsychiatric inventory questionnaire in individuals with dementia. J Am Geriatr Soc 2015;63:1448-52.

47 Giebel CM, Sutcliffe C, Stolt M, et al. Deterioration of basic activities of daily living and their impact on quality of life across different cognitive stages of dementia: a European study. Int. Psychogeriatr 2014;26:1283-93.

48 Howard $\mathrm{R}$, Phillips $\mathrm{P}$, Johnson $\mathrm{T}$, et al. Determining the minimum clinically important differences for outcomes in the domino trial. Int $J$ Geriatr Psychiatry 2011;26:812-7.

49 Liang Z, Zeger S. Longitudinal data analysis of continuous and discrete responses for prepost designs. The Indian Journal of Statistics 2000;62:134-48.

50 Angrist JD, Imbens GW, Rubin DB. Identification of causal effects using instrumental variables. J Am Stat Assoc 1996;91:444-55.

51 Harris PA, Taylor R, Thielke $\mathrm{R}$, et al. Research electronic data capture (REDCap)--a metadata-driven methodology and workflow process for providing translational research informatics support. J Biomed Inform 2009:42:377-81. 